\title{
An in-vitro assessment of the hydraulic characteristics of the Mark II Abrams-Lucas mitral valve prosthesis
}

\author{
J. T. M. WRIGHT
}

From the Bio-Engineering and Medical Physics Unit, University of Liverpool

Wright, J. T. M. (1977). Thorax, 32, 296-302. An in-vitro assessment of the hydraulic characteristics of the Mark II Abrams-Lucas mitral valve prosthesis. As a result of the durability problems associated with the first Abrams-Lucas mitral valve, a redesigned model has recently been introduced into limited clinical trials. The new valve was subjected to in-vitro pulsatile flow studies, and measurements were made of mean diastolic pressure gradient and volume of reflux on closure. Similar measurements were made on other mitral valve prostheses of comparable size. High-speed cinematography was used to analyse the motion of the occluder during the simulated cardiac cycle, and the flow patterns produced by the valve in the model ventricular cavity were observed and photographed.

The pressure gradient of the Abrams-Lucas valve was significantly lower than that of the $29 \mathrm{~mm}$ Björk-Shiley valve and all other prostheses tested, but its reflux level was higher at $12 \mathrm{ml}$ per stroke. The valve opened and closed smoothly and the flow visualisation study revealed that the valve produced a large vortex or swirl in the model ventricular cavity.

The first Abrams-Lucas mitral valve prosthesis underwent limited clinical trials a decade ago (Abrams, 1968) and proved to be haemodynamically satisfactory as a mitral valve replacement. The limited experience gained from the 40 or so surviving patients indicated that the incidence of thromboembolic complication associated with the prosthesis was appreciably less than that associated with the Starr-Edwards model 6120 silicone rubber ball valve which was inserted into a similar group of patients at the same centre (Abrams, 1976). However, the durability of the prosthesis was found to be inadequate because there was significant wear of the hooks which retained the flap to the orifice. This wear limited the useful life of the valve to five to seven years. Figure 1 shows an example of the typical wear pattern experienced with this early prosthesis. A further disadvantage of the valve was that the hook engagement was such that the valve could too easily be taken apart in vitro. However, the flap never became detached in practice until extreme hook wear had occurred.

Because of the high wear rate experienced in

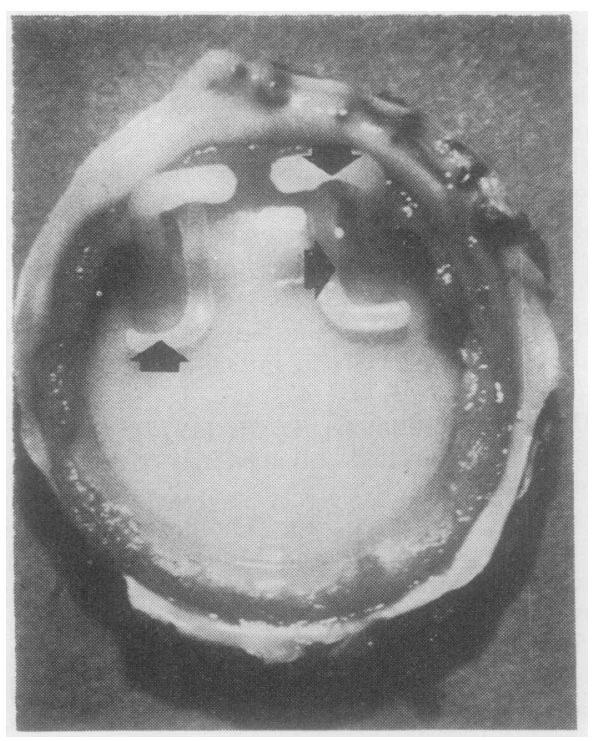

Fig. 1 An example of the hook wear (indicated by arrows) of the early Abrams-Lucas mitral valve (52 months). 
clinical use, an in-vitro study of the opening and closing action of the valve was made using highspeed cinematography, and the results were briefly reported (Wright, 1970). Accelerated wear and other durability studies were later undertaken by workers at the National Tribology Centre, Risley, and, using their recommendations, a new model of the Abrams-Lucas mitral valve prosthesis has recently been produced under a research contract with the Department of Health and Social Security. Like its predecessor the Mark II valve (shown in Fig. 2) is a simple flap type prosthesis. The flap is retained, and its range of movement controlled, by pairs of hooks attached to the orifice ring and the flap. The valve is injection-moulded from polypropylene, and the polypropylene felt suture ring is made an integral part of the valve orifice during the injection moulding process. A single size of prosthesis with a tissuemounting diameter of $30 \mathrm{~mm}$ is being manufactured at present. Limited clinical trials of the new valve are being carried out at three centres in the UK.

An independent study of the in-vitro hydraulic characteristics of this Mark II valve has been carried out, together with a high-speed cinematographic study of the valve action and observed flow patterns produced in a model ventricle. The results are presented in this paper and are compared with studies carried out on some comparable sized mitral valve prostheses in clinical use.

\section{Method}

The valves were mounted in the mitral position between the model left atrium and left ventricle

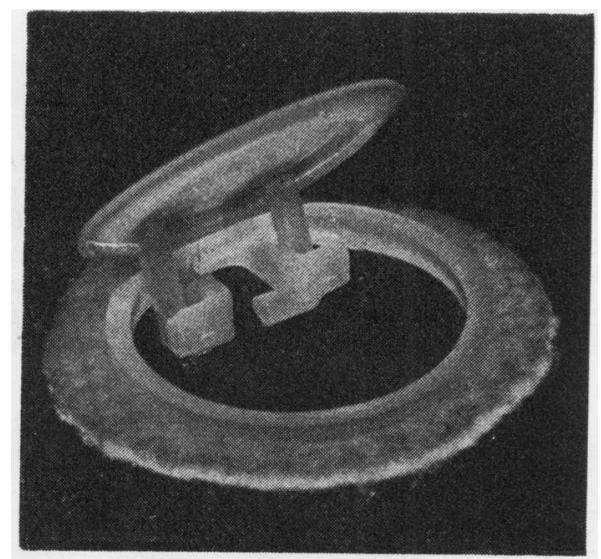

Fig. 2 The Mark II Abrams-Lucas mitral valve prosthesis. of a pulse duplicator and subjected to sinusoidally varying pulsatile flow. This was accomplished by moving the piston, which formed the left ventricular apex, back and forth in a sinusoidal manner in the ventricular cavity, which was cylindrical in shape. The stroke volume was fixed at $60.7 \mathrm{ml}$, but the pumping speed could be varied over the range 60-140 cycles per minute. During simulated diastole the test liquid (a water-glycerine mixture of $3 \mathrm{cP}$ at $37^{\circ} \mathrm{C}$ and $1.10 \times 10^{-3} \mathrm{~kg} \mathrm{~m}^{-3}$ density) was drawn through the valve under test. The instantaneous pressure drop, or gradient across the valve, was measured by a high-fidelity (flat to $40 \mathrm{~Hz}$ ) differential pressure transducer the output of which was electronically integrated over the diastolic period to obtain the mean diastolic gradient. The diastolic period was defined as the time from the first zero crossing point of the ventricular-atrial pressure difference to the point of zero flow velocity at end-diastole (a piston velocity transducer was used to define the latter point). During systole liquid was ejected from the aortic outflow tract via a pressurising valve, which caused the intraventricular systolic pressure to rise to an approximately normal level. The quantity of liquid ejected per stroke could be collected and compared with the known piston displacement to deduce the reflux past the prosthesis during closure and during systole.

The flow patterns produced in the ventricular cavity by the Abrams-Lucas mitral valve were observed by suspending a small quantity of white polystyrene beads (185-420 $\mu \mathrm{m}$ diameter) in the water-glycerine test liquid and shining a thin sheet of high-intensity light through the central axis of the ventricular chamber. The resulting flow patterns were recorded by a $35 \mathrm{~mm}$ single lens reflex camera. The camera shutter was electrically released by a cam-driven microswitch actuated from the test rig drive shaft, and the cam was adjusted so that photographs were taken at chosen portions of the simulated diastolic filling cycle. Full details of the techniques uset together with results from a number of aortic and mitral valve prostheses are given by Wright (1977).

\section{Results}

Figure 3 shows the variation of mean diastolic pressure gradient with mean diastolic flow rate. The latter was measured by dividing the stroke volume by the diastolic filling time. Also shown on this graph for comparison are the characteristics of the Lillehei-Kaster 25 and 22 valves (the numbers refer to the orifice diameters of this make 


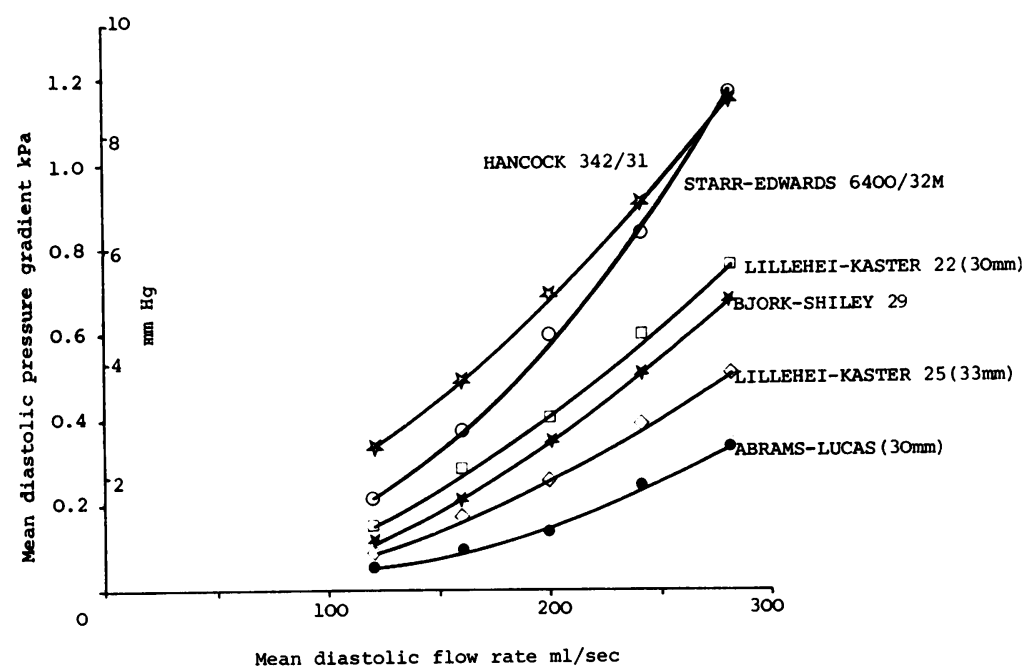

Fig. 3 Graph showing variation of mean diastolic gradient with change of mean diastolic flow rate.

of valve) and the Björk-Shiley $29 \mathrm{~mm}$ tilting disc valve, the Starr-Edwards 6400-32M tracked cage ball valve, and the Hancock $31 \mathrm{~mm}$ porcine bioprosthesis. The volume of reflux through the valves during closure and simulated systole is shown in Figure 4.

Frame by frame measurements made from the high-speed ciné film (taken at a camera speed of 200 frames per second and with a diastolic filling time of $375 \mathrm{~ms}$ ) allowed Fig. 5 to be drawn. This shows how the angle of the flap varied throughout the simulated cycle. The flow patterns produced by the valve during diastole are shown in Fig. 63 These photographs were taken with a shutte speed of $1 / 30 \mathrm{~s}$ at various times (shown on the photographs) after the onset of diastole.

\section{Discussion}

The pressure-gradient characteristics of the Abrams-Lucas valve were significantly better than those of all the other prostheses tested. For exo

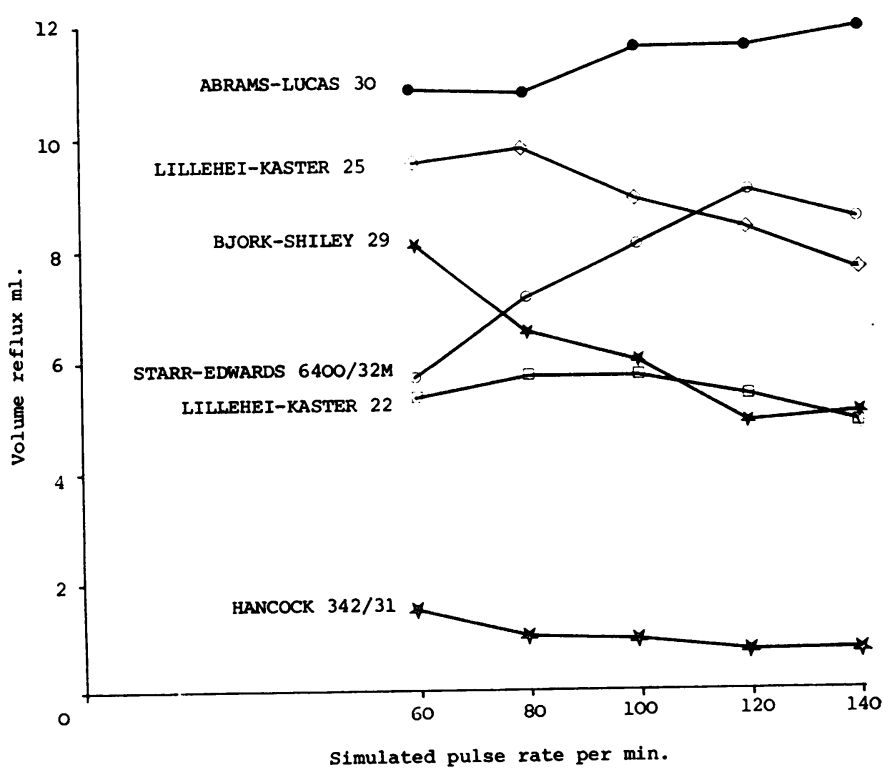

Fig. 4 Graph showing variation of volume reflux on closure and during systole with change of pulse rate. Test liquid was water-glycerine $\left(1 \cdot 10 \times 10^{-3}\right.$ $\left.\mathrm{kg} \mathrm{m}^{-3}, 3.0 \mathrm{cP}, 37^{\circ} \mathrm{C}\right)$. Ventricular section was at $45^{\circ}$ to horizontal plane with aorti६o outlet tract in the same horizontal plane as the mitral valve. Stroke volume was $ᄋ$ $60.7 \mathrm{ml}$. 


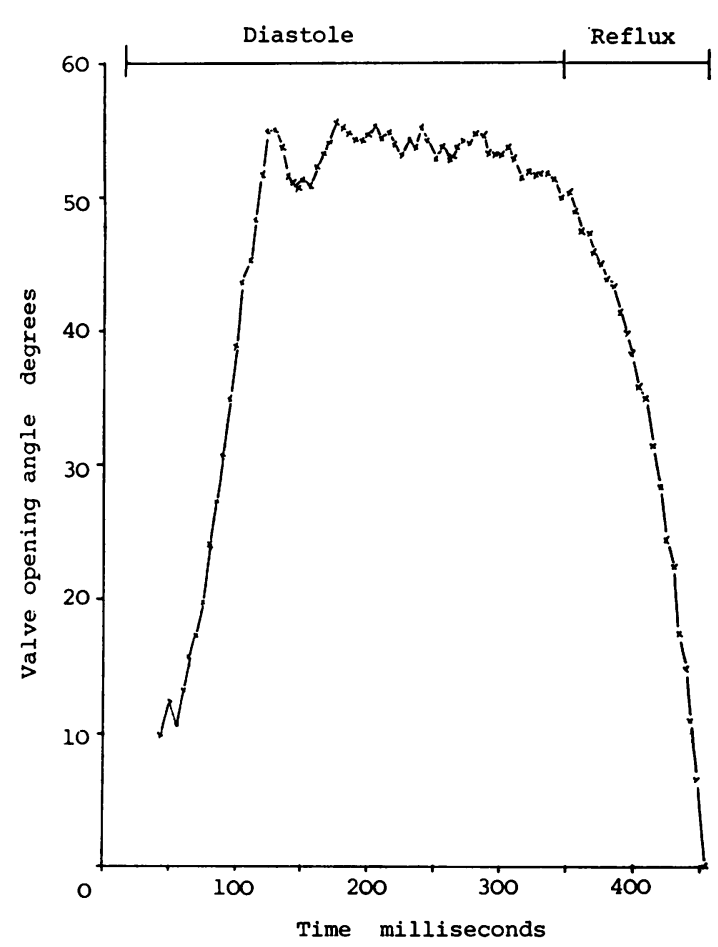

Fig. 5 Graph showing valve opening angle of flap of the Abrams-Lucas mitral valve. Measurements were made from consecutive frames of the highspeed ciné film. Camera speed 200 frames per second.

ample, under simulated exercise conditions the Björk-Shiley $29 \mathrm{~mm}$ valve caused approximately twice the gradient of the Abrams-Lucas valve.

However, the volume of reflux through the Abrams-Lucas valve was rather high at $11-12 \mathrm{ml}$ per stroke (representing $19.9 \%$ of the stroke volume of $60.7 \mathrm{ml}$ ). This was just below that level of insufficiency (20\%) which Kennedy et al. (1970) regarded as being clinically significant. In patients with stroke volumes greater than $61 \mathrm{ml}$ the percentage reflux would clearly be less than $19.9 \%$, but in patients with smaller stroke volumes the valve might be expected to produce clinically significant incompetence. This backflow occurred during the closing phase of the valve, not while the valve was shut. By contrast, the Björk-Shiley $29 \mathrm{~mm}$ valve had a small leakage path through the annular gap between the disc and the orifice ring. Thus some of the backflow did occur during the systolic part of the simulated cardiac cycle. It was for this reason that the Björk-Shiley valve displayed the comparatively higher levels of reflux at low pulse rates when the systolic period and hence the leakage time was longer. However, with sine wave flow function testing, the diastolic and systolic periods are always equal, while in the heart the systolic period at low pulse rates is significantly less than the diastolic period. Hence in the case of the Björk-Shiley valve the reflux level found at low pulse rates in these in-vitro tests would be expected to be somewhat higher than that experienced in vivo. At high pulse rates, when systole and diastole are comparable in length, no significant error would be expected. Another difference between physiological and sinewave testing concerns the rate of change of flow during early systole. It is usually the backflow through the valve during early systole which causes closure to occur. In vivo an abrupt contraction of the left ventricle might produce a greater rate of change of flow than occurred with a sinewave function. However, it will be seen from Fig. 4 that many valves showed constant or slightly diminished reflux as pulse rate, and hence rate of change of flow, was increased. I think that this difference would not significantly affect levels of reflux measured in these tests.

A more important variable affecting reflux values was the orientation of the ventricular chamber and the valve itself. All the prostheses, with the exception of the Hancock bioprosthesis, had occluders the densities of which were different from that of the test liquid. This was most marked in the tilting disc valves. Gravitational forces, therefore, could aid or oppose valve closure, depending on the mounted position of the valve. The angulation of the natural mitral ring varies, depending on the physical characteristics of the patient and on posture. By way of compromise these tests were therefore carried out with the mitral valve angled at $45^{\circ}$ to the vertical plane with the dummy aortic valve in the same horizontal plane. The tilting disc valves were then orientated as recommended by their leading proponents, ie, the Lillehei-Kaster opened towards the ventricular septum, and the Björk-Shiley opened away from it.

Insufficiency in a prosthesis will also cause an increase in gradient, for the heart will have to increase the volume pumped to compensate for the reflux past the prosthesis. To allow for this effect Fig. 7 has been constructed. It shows how the mean diastolic pressure gradient varied with reflux-corrected diastolic flow rate. Also shown is the approximate cardiac output. It can be seen that, in spite of its higher reflux level, the AbramsLucas valve led to a significantly lower pressure gradient than the other valves tested. It can also 

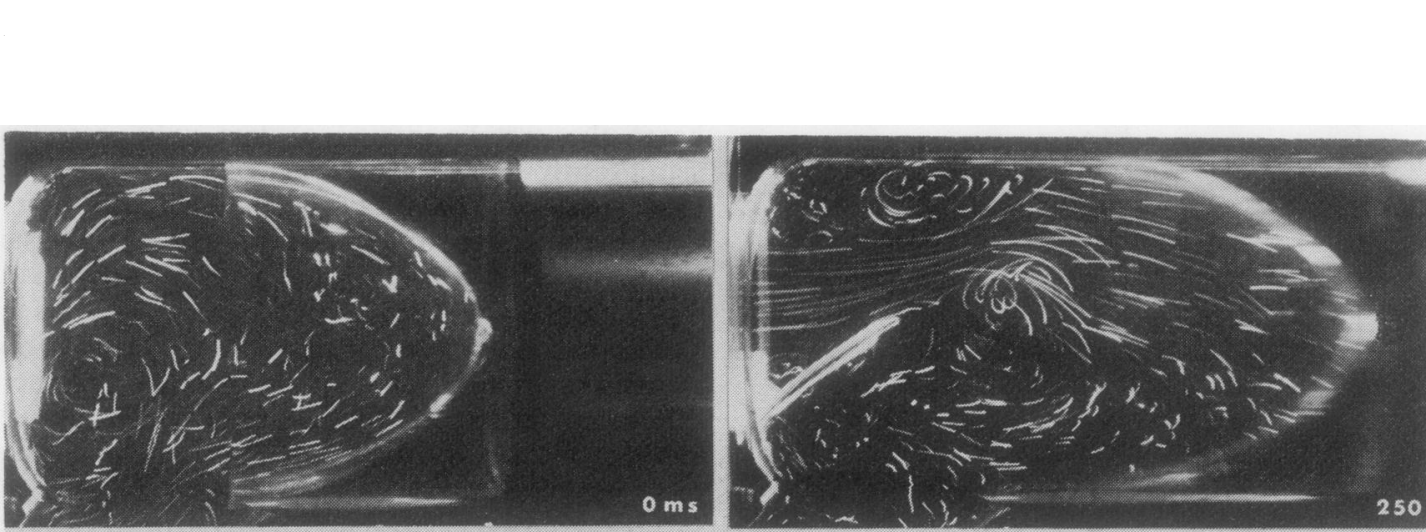

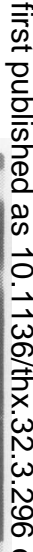
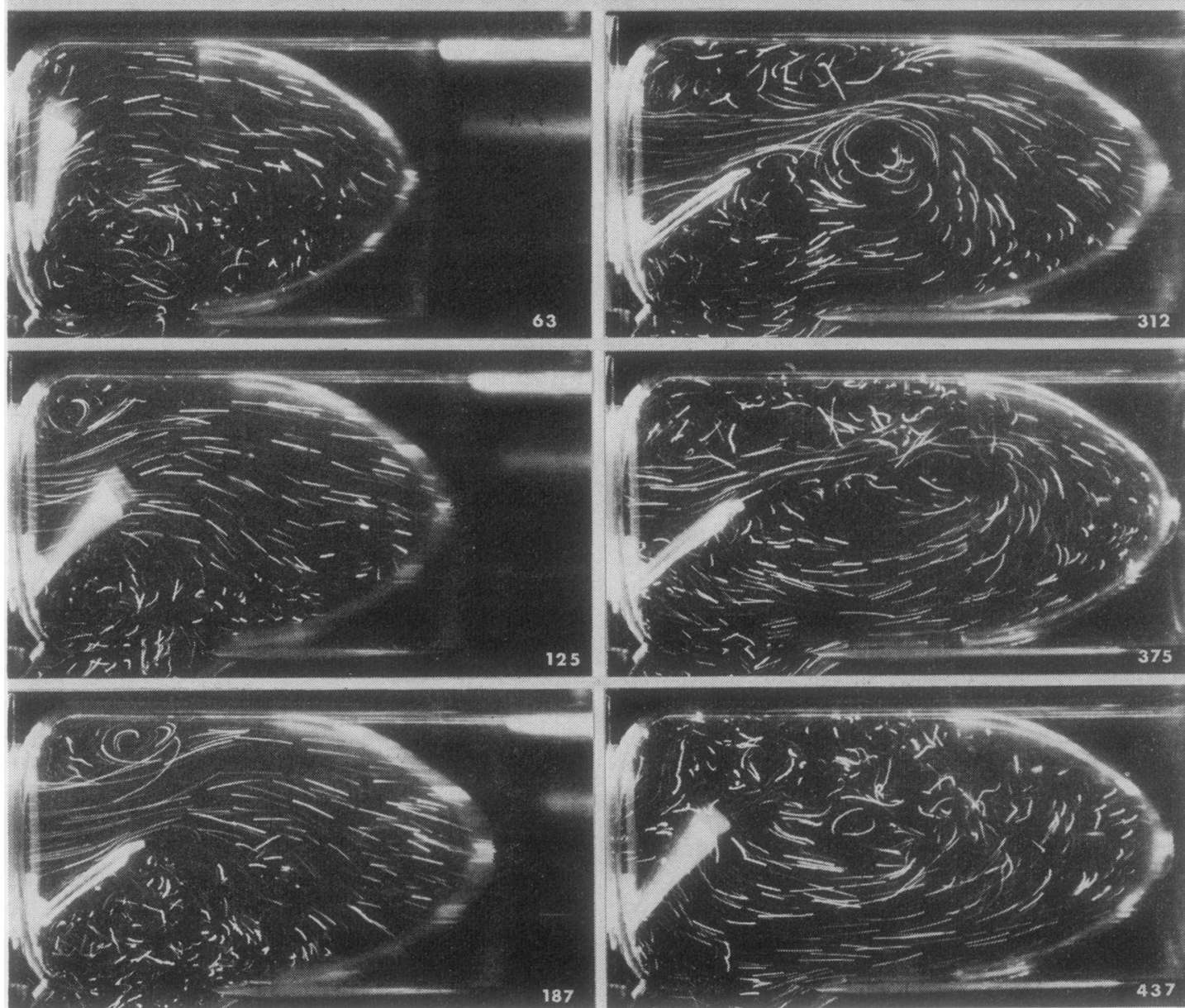

Fig. 6 Flow patterns produced by the Abrams-Lucas mitral valve in the model left ventricle. Numbers in the lower right-hand corner of each photograph show time (in milliseconds) after onset of diastole. Total length of diastole was $375 \mathrm{~ms}$. 


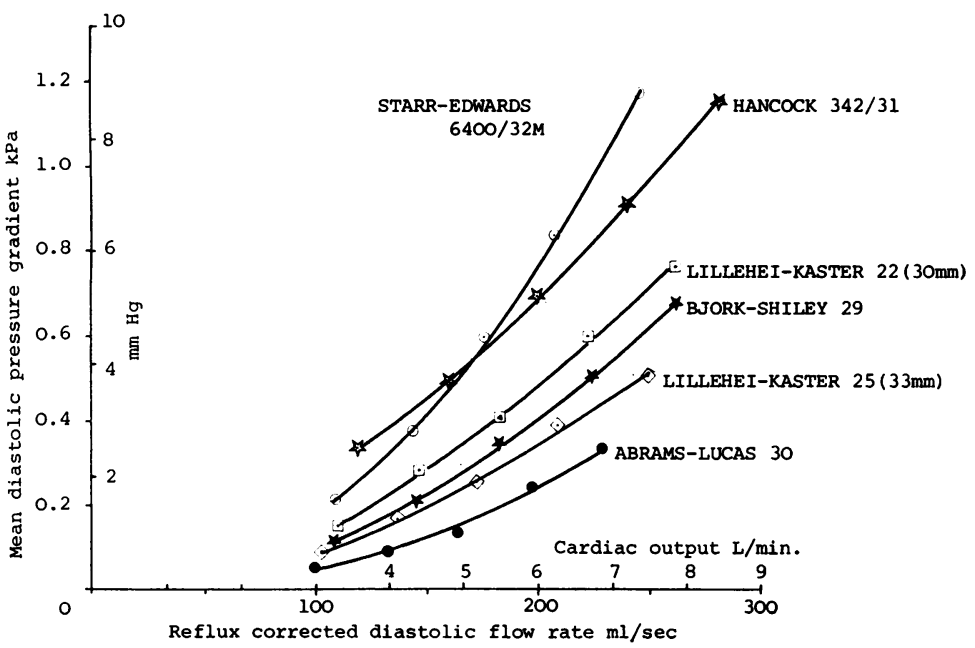

Fig. 7 Graph showing variation of mean diastolic pressure gradient with change of reflux-corrected mean diastolic flow rate. be seen that after correction the Hancock $31 \mathrm{~mm}$ prosthesis resulted in a lower pressure gradient than the Starr-Edwards 6400-32M tracked valve.

Analysis of the high-speed film showed that, on opening, the flap of the Abrams-Lucas valve initially moved parallel to the orifice ring until the hooks came to the end of their travel. The flap then moved through an arc until it reached the limits of its travel when, after a small rebound, it remained at an angle of $54^{\circ}$ to the orifice ring. Complete opening occurred in $130 \mathrm{~ms}$. Closure of the flap began $50 \mathrm{~ms}$ before the end of diastole, but as the ciné studies were carried out with the ventricular section upright (using water for the dye injection studies) the buoyancy of the flap would have produced a small gravitational force which might have aided early valve closure. Closure was complete $112 \mathrm{~ms}$ after end-diastole. The bead flow observation studies shown in Fig. 6 illustrate how the flow was distributed through and past the valve. The streak length - the distance that the bead had travelled during the period of the camera shutter opening (nominally $1 / 30 \mathrm{~s}$ )-was related to the velocity of the particle. These patterns give a qualitative indication of flow velocity rather than a quantitative measurement, for it cannot always be assumed that an individual particle was travelling at uniform velocity during the shutter opening, or had remained within the width of the light beam for the whole of that time. It can be seen from these patterns that small eddies were formed round the flap. Measurements taken directly from the cine film showed that some of these eddies rotated through $360^{\circ}$ in a circle of
$3 \mathrm{~mm}$ diameter during $1 / 60 \mathrm{~s}$. A weak but large vortex was produced in the left ventricular cavity by the end of the diastolic period.

\section{Conclusion}

The pressure gradient characteristics of the Abrams-Lucas valve prosthesis compare favourably with those of other mitral valve prostheses in clinical use. The reflux level was found to be higher than desirable, but this should not cause clinically significant insufficiency except in patients with low cardiac stroke volumes. The opening and closing characteristics were unremarkable, and the flow patterns produced by the valve in the model ventricle were similar to those produced by two tilting disc valves (Wright, 1977). The design features of the new valve should overcome two of the problems associated with the early model, for accelerated wear studies have shown the expected life to be at least five times that of the former valve (Abrams, 1976), and the thickened and reshaped hooks ensure adequate retention of the flap in the orifice.

Part of this work was carried out using equipment purchased on British Heart Foundation Grant no. 365. The generosity of the various manufacturers in donating prostheses is gratefully acknowledged. Mr. L. D. Abrams kindly provided a slide from which Fig. 1 was made.

\section{References}

Abrams, L. D. (1968). A mitral valve prosthesis. Pro- 
ceedings of Royal Society of Medicine, 61, 219.

Abrams, L. D. (1976). Personal communication.

Kennedy, J. W., Yarnall, S. R., Murray, J. A., and Figley, M. M. (1970). Quantitative angiocardiography. IV. Relationships of left atrial and ventricular pressure and volume in mitral valve disease. Circulation, 41, 817-824.

Wright, J. T. M. (1970). The flow characteristics of prosthetic mitral heart valves. $\mathrm{PhD}$ thesis, University of Liverpool.
Wright, J. T. M. (1977). A flow visualisation study of prosthetic aortic and mitral heart valves in $\mathbf{a}_{\square}$ model of the aorta and left heart. Engineering in Medicine, 6, No. 2.

Requests for reprints to: J. T. M. Wright, Bio- $\infty$ Engineering and Medical Physics Unit, Liverpool $\vec{\circ}$ University, Mount Pleasant, PO Box 147, LiverpoolL69 3BX. 